\title{
Squares of congruence subgroups of the extended modular group
}

\author{
Recep Sahin and Sebahattin Ikikardes
}




\title{
SQUARES OF CONGRUENCE SUBGROUPS OF THE EXTENDED MODULAR GROUP
}

\author{
RECEP SAHIN AND SEBAHATTIN IKIKARDES
}

Received 6 June, 2013

\begin{abstract}
In this paper, we generalize some results related to the congruence subgroups of modular group $\Gamma$, given in [7] and [6] by Kiming, Schütt, and Verrill, to the extended modular group $\Pi$.

2010 Mathematics Subject Classification: $11 \mathrm{~F} 06$
\end{abstract}

Keywords: modular group, extended modular group, principal congruence subgroup

\section{INTRODUCTION}

The modular group $\Gamma=P S L(2, \mathbb{Z})$ is the discrete subgroup of $P S L(2, \mathbb{R})$ generated by two linear fractional transformations

$$
T(z)=-\frac{1}{z} \quad \text { and } \quad S(z)=-\frac{1}{z+1} .
$$

Then modular group $\Gamma$ has a presentation

$$
\Gamma=<T, S \mid T^{2}=S^{3}=I>\cong \mathbb{Z}_{2} * \mathbb{Z}_{3} .
$$

The extended modular group $\Pi=P G L(2, \mathbb{Z})$ has been defined by adding the reflection $R(z)=1 / \bar{z}$ to the generators of the modular group $\Gamma$. The extended modular group $\Pi$ has a presentation, see [5],

$$
\Pi=<T, S, R \mid T^{2}=S^{3}=R^{2}=(R T)^{2}=(R S)^{2}=I>\cong D_{2} *_{\mathbb{Z}_{2}} D_{3} .
$$

Here $T, S$ and $R$ have matrix representations

$$
\left(\begin{array}{cc}
0 & -1 \\
1 & 0
\end{array}\right), \quad\left(\begin{array}{cc}
0 & -1 \\
1 & 1
\end{array}\right) \text { and }\left(\begin{array}{ll}
0 & 1 \\
1 & 0
\end{array}\right) \text {, }
$$

respectively (in this work, we identify each matrix $A$ in $G L(2, \mathbb{Z})$ with $-A$, so that they represent the same element of $P G L(2, \mathbb{Z})$ ). Thus the modular group $\Gamma=$ $P S L(2, \mathbb{Z})$ is a subgroup of index 2 in the extended modular group $\Pi$.

Let us define $\Pi^{m}$ as the subgroup generated by the $m^{\text {th }}$ powers of all elements of $\Pi$, for some positive integer $m$. The subgroup $\Pi^{m}$ is called the $m^{\text {th }}$ power subgroup of $\Pi$. As fully invariant subgroups, they are normal in $\Pi$. 
Then, power subgroups of the extended modular group $\Pi$ were examined by Sahin, Ikikardes and Koruoglu in [10]. The authors showed that

$$
\begin{aligned}
& \left|\Pi: \Pi^{2}\right|=4, \Pi^{2}=\Gamma^{2} \\
& \Pi^{2}=<S, T S T \mid(S)^{3}=(T S T)^{3}=I>\cong \mathbb{Z}_{3} * \mathbb{Z}_{3},
\end{aligned}
$$

Also, from [5], we have the following. Let $A=\left(\begin{array}{ll}a & b \\ c & d\end{array}\right)$ represent a general element of $\Pi$. For each integer $N \geq 1$, we define

$$
\begin{aligned}
& \Pi(N)=\{A \in \Pi \mid a \equiv d \equiv \pm 1 \text { and } b \equiv c \equiv 0(\bmod N)\}, \\
& \Gamma(N)=\Pi(N) \cap \Gamma .
\end{aligned}
$$

These are normal subgroups of finite index in $\Pi$, and they are called as the principal congruence subgroups. If $N>2$ then $\Pi(N)=\Gamma(N)$ and if $N=2$ then $\Pi(2) \geq$ $\Gamma(2) \geq \Pi(4)=\Gamma(4)$. A subgroup $K$ of $\Pi$ contains some $\Pi(N)$ if and only if it contains some $\Gamma(N)$. Such a subgroup $K$ is called a congruence subgroup, and the level of $K$ is the least $n$ such that $\Pi(N) \leq K$. Any other subgroup of finite index in $\Pi$ is called a non-congruence subgroup.

The most important of the congruence subgroups of $\Pi$ are

$$
\Pi_{0}(N)=\{A \in \Pi \mid c \equiv 0(\bmod N)\}
$$

and

$$
\Pi^{1}(N)=\{A \in \Pi \mid a \equiv d \equiv \pm 1 \text { and } c \equiv 0(\bmod N)\} .
$$

From [9], it is known that

$$
\Pi_{0}(N)=\Gamma_{0}(N) \cup T R . \Gamma_{0}(N) \text { and } \Pi^{1}(N)=\Gamma^{1}(N) \cup T R . \Gamma^{1}(N) .
$$

Also, it is clear that $\Pi^{1}(N) \triangleleft \Pi_{0}(N)$ and for $N>2,\left|\Pi_{0}(N): \Pi^{1}(N)\right|=\varphi(N) / 2$ where $\varphi$ is the Euler Phi function (for the index $\Gamma^{1}(N)$ in $\Gamma_{0}(N)$, see [4]).

On the other hand, in [7] and [6], Kiming Schütt, and Verrill studied lifts of projective congruence subgroups. Now, we recall the following information from [7]. For a subgroup $\Lambda$ of $S L(2, \mathbb{Z})$ denote by $\bar{\Lambda}$ the image of $\Lambda$ in $P S L(2, \mathbb{Z})$. A lift of $\bar{\Lambda}$ is a subgroup of $S L(2, \mathbb{Z})$ that projects to $\bar{\Lambda}$ in $P S L(2, \mathbb{Z})$. A lift is called a congruence lift if it is a congruence subgroup.

In [7] and [6], the authors gave some consequences of their main results for the groups generated by squares of elements in congruence subgroups. These results are

a) $\Gamma(N)^{2}$ is a congruence if and only if $N \leq 2$.

b) All lifts of $\Gamma_{0}(N) \leq P S L(2, \mathbb{Z})$ are congruence subgroups of $S L(2, \mathbb{Z})$ if and only if either $N \in\{3,4,8\}$ or if $4 \nmid N$ and all odd prime divisors of $N$ are congruent to 1 modulo 4 . 
c) All lifts of $\Gamma^{1}(N) \leq P S L(2, \mathbb{Z})$ are congruence subgroups of $S L(2, \mathbb{Z})$ if and only if $N \leq 4$.

The congruence and principal congruence subgroups (especially, $\Pi(2), \Gamma(2)$, $\Gamma_{0}(N)$ and $\left.\Gamma^{1}(N)\right)$ of $\Gamma$ and $\Pi$ have been studied from various aspects in the literature, for example, number theory, modular forms, modular curves, Belyi's theory, graph theory, (please see [1], [2], [3] and [8]).

In this paper, we generalize the above results related with congruence subgroups of $\Gamma$, given in [7] and [6], to the extended modular group $\Pi$.

\section{Squares of Congruence Subgroups of $\Pi$}

From [5], if $N>2$ then $\Pi(N)=\Gamma(N)$ and so $\Pi(N)^{2}=\Gamma(N)^{2}$. Thus, if $N>2$ then $\Pi^{2}(N)$ is not a congruence. Also, from [10] and [5], $\Pi^{2}(1)=\Pi^{\prime}$ and $\Pi(6) \leq$ $\Pi^{2}(1)$ and so $\Pi^{2}(1)$ is a congruence subgroup. Therefore we need the following theorem.

Theorem 1. $\Pi(2)^{2}=\Pi(4)$.

Proof. We know that the group structure of $\Pi(2)$ is

$$
\begin{aligned}
\Pi(2) & =<T R, R S T S, R S^{2} T S^{2} \mid(T R)^{2}=(R S T S)^{2}=\left(R S^{2} T S^{2}\right)^{2}=I> \\
& \cong \mathbb{Z}_{2} * \mathbb{Z}_{2} * \mathbb{Z}_{2} .
\end{aligned}
$$

Let $a=T R, b=R S T S, c=R S^{2} T S^{2}$. Then the quotient group $\Pi(2) / \Pi(2)^{2}$ is the group obtained by adding the relation $X^{2}=I$ for all $X \in \Pi(2)$ to the relations of $\Pi(2)$. Thus we have

$$
\Pi(2) / \Pi(2)^{2} \cong<a, b, c \mid a^{2}=b^{2}=c^{2}=(a b)^{2}=(a c)^{2}=(b c)^{2}=\ldots=I>\text {. }
$$

As $a^{2}=b^{2}=c^{2}=I$, we obtain

$$
\Pi(2) / \Pi(2)^{2} \cong \mathbb{Z}_{2} \times \mathbb{Z}_{2} \times \mathbb{Z}_{2}
$$

Therefore, we obtain $\left|\Pi(2): \Pi(2)^{2}\right|=8$.

Thus we use the Reidemeister-Schreier process to find the presentation of the subgroup $\Pi(2)^{2}$. Now we choose $\Sigma=\{I, a, b, c, a b, a c, b c, a b c\}$ as a Schreier transversal for $\Pi(2)^{2}$. According to the Reidemeister-Schreier method, we can form all possible products :

$$
\begin{aligned}
& \text { I.a. }(a)^{-1}=I \text {, } \\
& \text { a.a. }(I)^{-1}=I \text {, } \\
& \text { I.b. }(b)^{-1}=I \text {, } \\
& \text { a.b. }(a b)^{-1}=I \text {, } \\
& \text { b.a. }(a b)^{-1}=b a b a \text {, } \\
& \text { b.b. }(I)^{-1}=I \text {, } \\
& \text { c.a. }(a c)^{-1}=c a c a \text {, } \\
& \text { c.b. }(b c)^{-1}=c b c b \text {, } \\
& \text { ab.a. }(b)^{-1}=a b a b, \\
& a b \cdot b \cdot(a)^{-1}=I \text {, } \\
& \text { I.c. }(c)^{-1}=I \text {, } \\
& \text { a.c. }(a c)^{-1}=I \text {, } \\
& \text { b.c. }(b c)^{-1}=I \text {, } \\
& \text { c.c. }(I)^{-1}=I \text {, } \\
& a c . a \cdot(c)^{-1}=a c a c, \\
& a c . b \cdot(a b c)^{-1}=a c b c b a \text {, } \\
& \text { ab.c. }(a b c)^{-1}=I \text {, } \\
& \text { bc.a. }(a b c)^{-1}=b c a c b a, \quad b c . b .(c)^{-1}=b c b c, \quad b c . c .(b)^{-1}=I, \\
& \text { ac.c. }(a)^{-1}=I \text {, } \\
& a b c \cdot a \cdot(b c)^{-1}=a b c a c b, \quad a b c \cdot b \cdot(a c)^{-1}=a b c b c a, \\
& \text { abc.c. }(a b)^{-1}=I \text {, }
\end{aligned}
$$


as $a^{-1}=a, b^{-1}=b$, and $c^{-1}=c$. Also, since $(b a b a)^{-1}=a b a b,(c a c a)^{-1}=$ $a c a c,(c b c b)^{-1}=b c b c,(b c a c b a)^{-1}=a b c a c b$ and $(a c b c b a)^{-1}=a b c b c a$, the generators of $\Pi(2)^{2}$ are $a b a b=\left(\begin{array}{ll}1 & 4 \\ 0 & 1\end{array}\right)$, acac $=\left(\begin{array}{ll}1 & 0 \\ 4 & 1\end{array}\right)$,

$b c b c=\left(\begin{array}{cc}5 & 4 \\ -4 & -3\end{array}\right), a b c a c b=\left(\begin{array}{cc}-7 & -12 \\ -4 & -7\end{array}\right)$ and $a b c b c a=\left(\begin{array}{cc}5 & -4 \\ 4 & -3\end{array}\right)$.

From [7, Lemma 32], $\Pi(2)^{2}=\Gamma(4)$. As $\Gamma(4)=\Pi(4)$, we obtain $\Pi(2)^{2}=\Pi(4)$.

Using the above results, we have the following.

Proposition 1. $\Pi(N)^{2}$ is a congruence if and only if $N \leq 2$.

Now we present some results related with the congruence subgroups $\Pi_{0}(N)$ and $\Pi^{1}(N)$ of $\Pi$. To do this, we suppose that

$$
A=\left(\begin{array}{cc}
x & * \\
0 & x^{-1}
\end{array}\right)(\bmod N)
$$

is an element of $\Gamma_{0}(N)$. Then

$$
\text { TR.A }=\left(\begin{array}{cc}
-1 & 0 \\
0 & 1
\end{array}\right)\left(\begin{array}{cc}
x & * \\
0 & x^{-1}
\end{array}\right)=\left(\begin{array}{cc}
-x & * \\
0 & x^{-1}
\end{array}\right)(\bmod N)
$$

is an element of $\Pi_{0}(N)$. Therefore

$$
(T R A)^{2}=\left(\begin{array}{cc}
-x & * \\
0 & x^{-1}
\end{array}\right)\left(\begin{array}{cc}
-x & * \\
0 & x^{-1}
\end{array}\right)=\left(\begin{array}{cc}
x^{2} & * \\
0 & x^{-2}
\end{array}\right)(\bmod N)
$$

is an element of $\Gamma_{0}(N)^{2}$. Thus, we get $\Pi_{0}(N)^{2}=\Gamma_{0}(N)^{2}$.

Similarly to the case $\Pi_{0}(N)$, if

$$
B=\left(\begin{array}{cc}
1 & * \\
0 & 1
\end{array}\right)(\bmod N)
$$

is an element of $\Gamma^{1}(N)$, then

$$
\text { TR.B }=\left(\begin{array}{cc}
-1 & 0 \\
0 & 1
\end{array}\right)\left(\begin{array}{ll}
1 & * \\
0 & 1
\end{array}\right)=\left(\begin{array}{cc}
-1 & * \\
0 & 1
\end{array}\right)(\bmod N)
$$

is an element of $\Pi^{1}(N)$. Therefore

$$
(T R B)^{2}=\left(\begin{array}{cc}
-1 & * \\
0 & 1
\end{array}\right)\left(\begin{array}{cc}
-1 & * \\
0 & 1
\end{array}\right)=\left(\begin{array}{ll}
1 & * \\
0 & 1
\end{array}\right)(\bmod N)
$$

is an element of $\Gamma^{1}(N)^{2}$ and so we obtain $\Pi^{1}(N)^{2}=\Gamma^{1}(N)^{2}$.

On the other hand, if $\Pi_{0}(N)$ and $\Pi^{1}(N)$ are not congruence, then $\Pi_{0}(N)^{2}$ and $\Pi^{1}(N)^{2}$ are not congruence, since any lift of $\Pi_{0}(N)\left(\right.$ or $\left.\Pi^{1}(N)\right)$ necessarily contains $\Pi_{0}(N)^{2}$ (or $\left.\Pi^{1}(N)^{2}\right)$, from [7, Lemma 5]. Consequently, we have the following. 
Corollary 1. a) $\Pi_{0}(N)^{2}$ is not congruence if and only if either $N \notin\{3,4,8\}$ or if $4 \mid N$ and all odd prime divisors of $N$ are congruent to 3 modulo 4.

b) $\Pi^{1}(N)^{2}$ is not congruence if and only if $N>4$.

\section{REFERENCES}

[1] M. C. N. Cheng and A. Dabholkar, "Borcherds-Kac-Moody symmetry of $\mathcal{N}=4$ dyons," Commun. Number Theory Phys., vol. 3, no. 1, pp. 59-110, 2009.

[2] W. M. Goldman and W. D. Neumann, "Homological action of the modular group on some cubic moduli spaces," Math. Res. Lett., vol. 12, no. 4, pp. 575-591, 2005.

[3] W. J. Harvey, "Teichmüller spaces, triangle groups and Grothendieck dessins," in Handbook of Teichmüller theory. Volume I, ser. IRMA Lectures in Mathematics and Theoretical Physics, A. Papadopoulos, Ed. Zürich: European Mathematical Society (EMS), 2007, vol. 11, pp. 249-292.

[4] I. Ivrissimtzis and D. Singerman, "Regular maps and principal congruence subgroups of Hecke groups," Eur. J. Comb., vol. 26, no. 3-4, pp. 437-456, 2005.

[5] G. A. Jones and J. S. Thornton, "Automorphisms and congruence subgroups of the extended modular group,” J. Lond. Math. Soc., II. Ser., vol. 34, pp. 26-40, 1986.

[6] I. Kiming, "Lifts of projective congruence groups ii," Proc. Amer. Math. Soc., to appear.

[7] I. Kiming, M. Schütt, and H. A. Verrill, "Lifts of projective congruence groups," J. Lond. Math. Soc., II. Ser., vol. 83, no. 1, pp. 96-120, 2011.

[8] B. Köck and D. Singerman, "Real Belyi theory," Q. J. Math., vol. 58, no. 4, pp. 463-478, 2007.

[9] R. S. Kulkarni, "An arithmetic-geometric method in the study of the subgroups of the modular group," Am. J. Math., vol. 113, no. 6, pp. 1053-1133, 1991.

[10] R. Şahin, S. İkikardeş, and O. Koruoğlu, "On the power subgroups of the extended modular group $\bar{\Gamma}$, , Turk. J. Math., vol. 28, no. 2, pp. 143-151, 2004.

\section{Authors' addresses}

\section{Recep Sahin}

Balikesir University, Department of Mathematics, Cagis Kampusu, 10145 Balikesir, Turkey

E-mail address: rsahinebalikesir.edu.tr

\section{Sebahattin Ikikardes}

Balikesir University, Department of Mathematics, Cagis Kampusu, 10145 Balikesir, Turkey

E-mail address: skardes@balikesir.edu.tr 